\title{
Simulation of a Tubular Linear Magnetic Gear Using HTS Bulks for Field Modulation
}

\author{
Wenlong Li, K. T. Chau, and Jiangui Li
}

\begin{abstract}
A tubular linear magnetic gear is proposed, which newly utilizes high temperature superconductor (HTS) bulks for its field modulation. Compared to the conventional one, the proposed topology can significantly reduce the leakage flux, hence improving its force transmission capacity. By using the magnetic circuit modeling, the analytical formulae of air-gap flux density and thrust force are firstly derived. Then, the finite element method (FEM) is employed to validate the performance of the proposed linear magnetic gear.
\end{abstract}

Index Terms-FEM, field modulation, HTS bulk, linear magnetic gear, magnetic circuit.

\section{INTRODUCTION}

C OAXIAL magnetic gears are becoming attractive in some niche areas such as electric vehicle propulsion and wind power generation [1], [2], since they take some distinct advantages over the mechanical ones, such as higher efficiency, higher reliability, lower acoustic noise, inherent overload protection, and free from maintenance. Moreover, they are readily integrated into various electric machines, hence creating the so-called geared machines which can simultaneously offer high-frequency machine design and low-speed rotating motion. Furthermore, the concept of coaxial magnetic gears has been extended to the linear morphology [3] so as to improve the force capability of a linear motor.

The key of coaxial magnetic gears is the ferromagnetic segments which locate between the inner rotor and the outer rotor. When the sum of the outer-rotor PM pole-pair number $N_{1}$ and the inner-rotor PM pole-pair number $N_{2}$ equals the number of ferromagnetic segments $N_{s}$, torque transmission between the inner rotor and the outer rotor can be achieved without any mechanical contacts [4], [5]. The linear magnetic gear, flat or tubular, has a similar structure as its rotational counterpart. As shown in Fig. 1, the tubular linear magnetic gear consists of three parts: the low-speed mover, the high-speed mover and the stationary field-modulation ferromagnetic rings. Due to principle of field modulation, the two movers with different PM pole-pair numbers interact with one another to achieve force transmission. However, because of the finite permeance of ferromagnetic rings, it suffers from severe flux leakage, which significantly deteriorates the whole performance.

Manuscript received August 03, 2010; accepted September 17, 2010. Date of publication October 28, 2010; date of current version May 27, 2011. This work was supported by a Grant (HKU 710710E) from the Research Grants Council, Hong Kong Special Administrative Region, China.

The authors are with the Department of Electrical and Electronic Engineering, The University of Hong Kong, Hong Kong (e-mail: wlli@eee.hku.hk).

Color versions of one or more of the figures in this paper are available online at http://ieeexplore.ieee.org.

Digital Object Identifier 10.1109/TASC.2010.2080255

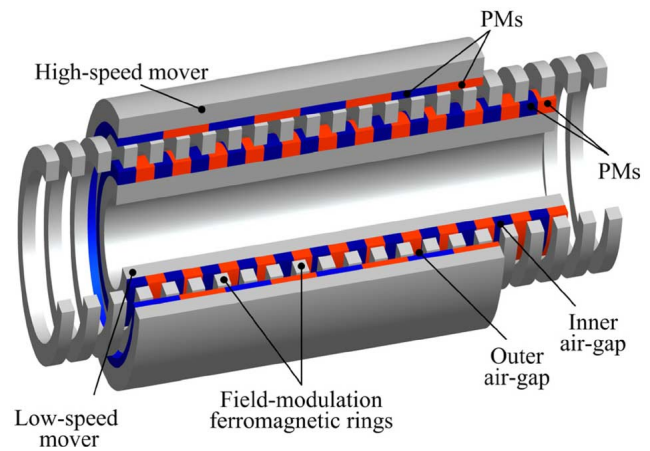

Fig. 1. Structure of conventional tubular linear magnetic gear.

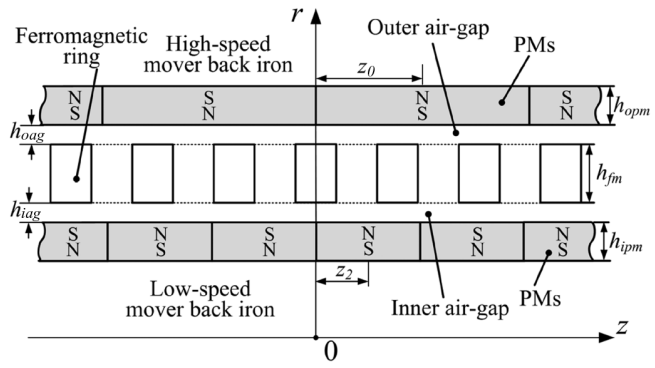

Fig. 2. Model of conventional tubular linear magnetic gear in cylindrical polar coordinates.

The purpose of this paper is to newly utilize high temperature superconducting (HTS) bulks [6], [7] to shield the flux leakage between the ferromagnetic rings for field modulation. Hence, the resulting HTS tubular linear magnetic gear can achieve high force transmission capacity. Both analytical formulation and finite element analysis will be given to validate the performance of the proposed magnetic gear.

\section{MATHEmatical Modeling}

In order to derive the analytical model, some assumptions are made: the permeability of the back irons of two movers and the ferromagnetic rings is assumed to be infinite, the permeability of the PMs is assumed to be equal to that of air, and the magnetic field only varies in the longitudinal direction. Due to the Meissner effect of HTS materials, the use of HTS bulks can force all PM flux passing through the ferromagnetic rings, thus significantly decreasing the flux leakage. So, the relative permeability of HTS bulks is also assumed to be zero.

In this modeling, the one-dimensional (1-D) path is adopted so that the flux goes straightly up and down and close at the infinite distance [6]. Fig. 2 shows the model of the tubular 


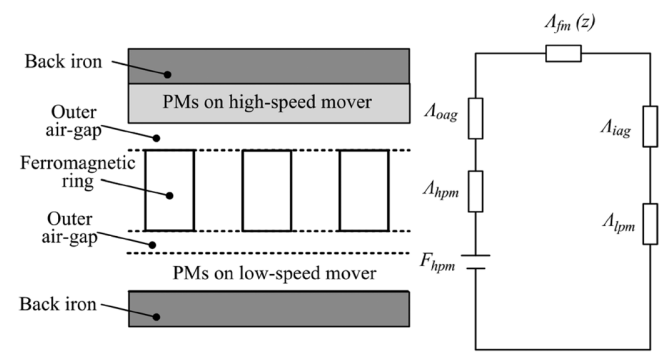

Fig. 3. Magnetic circuit excited by PMs on high-speed mover.

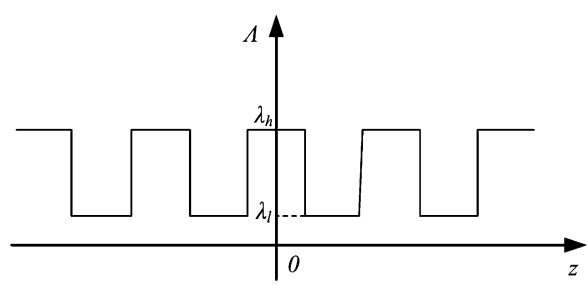

Fig. 4. Magnetic permeance waveform.

linear magnetic gear in the two-dimensional (2-D) cylindrical polar coordinates. Based on the aforementioned assumptions, the magnetic circuit can be considered as linear so that the resultant magnetic field can be treated as the superposition of the fields separately excited by PMs on the two movers. Fig. 3 shows the equivalent magnetic circuit when excited by PMs on the high-speed mover only. Thus, the equivalent total magnetic permeance in the longitudinal direction can be expressed as:

$$
\frac{1}{\Lambda(z)}=\frac{1}{\Lambda_{h p m}}+\frac{1}{\Lambda_{o a g}}+\frac{1}{\Lambda_{f m}(z)}+\frac{1}{\Lambda_{i a g}}+\frac{1}{\Lambda_{l p m}}
$$

where $\Lambda_{h p m}=\mu_{0} / h_{h p m}, \Lambda_{\text {oag }}=\mu_{0} / h_{\text {oag }}, \Lambda_{\text {iag }}=\mu_{0} / h_{\text {iag }}$, and $\Lambda_{l p m}=\mu_{0} / h_{l p m}$ are the magnetic permeances in the longitudinal direction of the PMs on the high-speed mover, outer air-gap, inner air-gap and PMs on the lsow-speed mover, respectively; $\Lambda_{f m}(z)$ is the magnetic permeance in the longitudinal direction of the field modulation segment area which is a function of the axial position $z$; and $h_{h p m}, h_{o a g}, h_{i a g}, h_{l p m}$ and $h_{f m}$ are the longitudinal lengths of PMs on the high-speed mover, outer air-gap, inner air-gap, PMs on the low-speed mover and ferromagnetic ring, respectively. When the segment area is the ferromagnetic ring, the corresponding $\Lambda_{f m}(z)$ is infinite. On the contrary, when the segment area is air space, $\Lambda_{f m}(z)=\mu_{0} / h_{f m}$; and when it is the HTS bulk, $\Lambda_{f m}(z)=0$.

Fig. 4 shows the magnetic permeance waveform of the equivalent magnetic circuit of a conventional linear magnetic gear. It can be resolved into a Fourier series:

$$
\Lambda(z)=\lambda_{0}+\sum_{m=1}^{\infty} \lambda_{m} \cos \left(m N_{s} \frac{2 \pi}{L} z\right)
$$

where $\lambda_{0}$ is the DC offset of the total equivalent magnetic permeance, $\lambda_{m}$ is the amplitude of the $m$ th harmonic magnetic permeance, $N_{s}$ is the number of ferromagnetic rings, and $L$ is the active length of the linear magnetic gear which is also equal to the total length of the high-speed mover.
The magnetomotive force (MMF) of PMs on the high-speed mover can also be expressed in a Fourier series:

$$
\begin{aligned}
F_{h p m}(z) & =\sum_{n, \text { odd }}^{\infty} \frac{4}{n \pi} H_{h c} h_{h p m} \cos \left(n N_{h p m} \frac{2 \pi}{L} z_{1}\right) \\
& =\sum_{n, \text { odd }}^{\infty} \frac{4}{n \pi} H_{h c} h_{h p m} \cos \left[n N_{h p m} \frac{2 \pi}{L}\left(z-z_{0}\right)\right]
\end{aligned}
$$

where $H_{h c}$ is the coercive force of PMs on the high-speed mover, $h_{h p m}$ is the magnet thickness, $N_{h p m}$ is the number of PM pole-pairs on the high-speed mover, $z_{1}$ is the axial position of PMs on the high-speed mover with respect to $z_{0}$, and $z_{0}$ is the corresponding initial position as shown in Fig. 2. Thus, the magnetic flux density excited by PMs on the high-speed mover can be calculated:

$$
\begin{aligned}
B_{h p m} & \\
= & F_{h p m}(z) \cdot \Lambda(z) \\
= & \left\{\frac{4}{\pi} H_{h c} h_{h p m} \cos \left[N_{h p m} \frac{2 \pi}{L}\left(z-z_{0}\right)\right]\right\} \\
& \times\left[\lambda_{0}+\lambda_{1} \cos \left(N_{s} \frac{2 \pi}{L} z\right)\right] \\
= & \frac{4}{\pi} H_{h c} h_{h p m} \lambda_{0} \cos \left[N_{h p m} \frac{2 \pi}{L}\left(z-z_{0}\right)\right] \\
& +\frac{2}{\pi} H_{h c} h_{h p m} \lambda_{1} \cos \left[\left(N_{h p m}+N_{s}\right) \frac{2 \pi}{L} z-N_{h p m} \frac{2 \pi}{L} z_{0}\right] \\
& +\frac{2}{\pi} H_{h c} h_{h p m} \lambda_{1} \cos \left[\left(N_{s}-N_{h p m}\right) \frac{2 \pi}{L} z+N_{h p m} \frac{2 \pi}{L} z_{0}\right] \\
= & B_{h p m}^{1}+B_{h p m}^{2}+B_{h p m}^{3}
\end{aligned}
$$

where $B_{h p m}^{1}$ has the same pole-pair number with that of PMs on the high-speed mover, and $B_{h p m}^{3}$ has the same pole-pair number with that of PMs on the low-speed mover. Thus, a thrust force can be produced by $B_{h p m}^{3}$ and PMs on the low-speed mover.

In order to obtain the expression of the thrust force, an equivalent current sheet is used to substitute the MMF of PMs on the low-speed mover. The fundamental MMF component of PMs on the low-speed mover is given by:

$$
F_{l p m 1}(z)=\frac{4}{\pi} H_{l c} h_{l p m} \cos \left[N_{l p m} \frac{2 \pi}{L}\left(z-z_{2}\right)\right]
$$

where $H_{l c}, h_{l p m}$ and $N_{l p m}$ are the coercive force, thickness and pole-pair number of PMs on the low-speed mover, and $z_{2}$ is its initial position as shown in Fig. 2. Thus, the corresponding equivalent current sheet is given by:

$$
I_{l p m}(z)=\frac{4}{\pi} H_{l c} h_{l p m} \sin \left[N_{l p m} \frac{2 \pi}{L}\left(z-z_{2}\right)\right]
$$

Consequently, by using Lorentz force law, the thrust force exerted on the low-speed mover can be obtained by:

$$
\begin{aligned}
F_{l m}(\theta) & =\int_{-L / 2}^{L / 2} \pi D_{l} B_{h p m}^{3} I_{l p m} d z \\
& \approx \frac{8}{\pi} N_{l p m} \lambda_{1} D_{l} H_{h c} H_{l c} h_{h p m} h_{l p m} \cos \theta
\end{aligned}
$$


where $\theta$ is the angular displacement between the centers of PMs of the two movers, hence written as $\theta=2 N_{h p m} \pi z_{0} / L+$ $2 N_{l p m} \pi z_{2} / L$, and $D_{l}$ is the diameter of the low-speed mover. The maximum thrust force occurs at $\theta$ equal to zero:

$$
F_{l m \_M a x}=\frac{8}{\pi} N_{l p m} \lambda_{1} D_{l} H_{h c} H_{l c} h_{h p m} h_{l p m}
$$

By using the same derivation, the magnetic flux density due to PMs on the low-speed mover can be expressed as:

$$
B_{l p m}^{3}=\frac{2}{\pi} H_{l c} h_{l p m} \lambda_{1} \cos \left(N_{h p m} \frac{2 \pi}{L} z+N_{h p m} \frac{2 \pi}{L} z_{2}\right)
$$

Then, the thrust force exerted on the high-speed mover can be obtained as:

$$
\begin{aligned}
F_{h m}(\theta) & =\int_{-L / 2}^{L / 2} \pi D_{h} B_{l p m}^{3} I_{h p m} d z \\
& \approx \frac{8}{\pi} N_{h p m} \lambda_{1} D_{h} H_{h c} H_{l c} h_{h p m} h_{l p m} \cos \theta
\end{aligned}
$$

where $D_{h}$ is the diameter of the high-speed mover.

From (8), it can be found that the developed thrust force is directly proportional to $\lambda_{1}$, namely the fundamental harmonic of the magnetic permeance of the equivalent magnetic circuit, which is governed by the field-modulation segment area. As shown in Fig. 4, the longitudinal magnetic permeance waveform of a conventional linear magnetic gear is a symmetrical square wave. By using Fourier analysis, the analytical formula $\lambda_{1}$ can be expressed as:

$$
\lambda_{1}=\frac{2\left(\lambda_{h}-\lambda_{l}\right)}{\pi} \sin \frac{\tau_{h} \pi}{\tau_{f m}}
$$

where $\lambda_{h}=\mu_{0} /\left(h_{h p m}+h_{\text {oag }}+h_{\text {iag }}+h_{\text {lpm }}\right), \lambda_{l}=\mu_{0} /\left(h_{f m}+\right.$ $\left.h_{\text {hpm }}+h_{\text {oag }}+h_{\text {iag }}+h_{\text {lpm }}\right), \tau_{h}$ is the length of ferromagnetic ring in $z$ direction, and $\tau_{f m}$ is the pole-pitch of ferromagnetic ring. When $\tau_{h}$ equals one half of $\tau_{f m}, \lambda_{1}$ achieves the peak value which is $2\left(\lambda_{h}-\lambda_{l}\right) / \pi$.

In this analytical model, the HTS bulks are considered to be an ideal superconductor, in which the magnetic field is totally ejected. Thus, the corresponding permeability is zero so that the value of $\lambda_{l}$ becomes zero. Consequently, it yields $\lambda_{1}=2 \lambda_{h} / \pi$, which physically means that the developed thrust forces of both movers can be improved from $\lambda_{1}=2\left(\lambda_{h}-\lambda_{l}\right) / \pi$ to $\lambda_{1}=$ $2 \lambda_{h} / \pi$. This theoretical improvement is then verified by applying finite element analysis to simulate the thrust forces of the proposed tubular linear magnetic gear with and without using HTS bulks.

\section{Finite ElEment ANAlysis}

The finite element method (FEM) is employed for analysis. In order to take into account magnetic saturation during analysis, the permeability of back irons and ferromagnetic rings is based on practical data of iron materials. On the other hand, the permeability of PMs is a constant based on the Nd-Fe-B material, while the permeability of HTS bulks is set to zero.

The key dimensions of the proposed gear are compared with the conventional one as listed in Table I. The field-modulation segments of the proposed gear adopts the zebra-striped design,
TABLE I

MACHINE PARAMETERS

\begin{tabular}{lll}
\hline \hline & Conventional & Proposed \\
\hline$N_{s}$ & 21 & 21 \\
$N_{h p m}$ & 6 & 6 \\
$N_{l p m}$ & 15 & 15 \\
$L$ & $264 \mathrm{~mm}$ & $264 \mathrm{~mm}$ \\
PM thickness & $4 \mathrm{~mm}$ & $4 \mathrm{~mm}$ \\
$D_{l}$ & $60 \mathrm{~mm}$ & $60 \mathrm{~mm}$ \\
$D_{h}$ & $96 \mathrm{~mm}$ & $90 \mathrm{~mm}$ \\
$h_{f m}$ & $6 \mathrm{~mm}$ & $3 \mathrm{~mm}$ \\
HTS height & N/A & $3 \mathrm{~mm}$ \\
\hline \hline
\end{tabular}

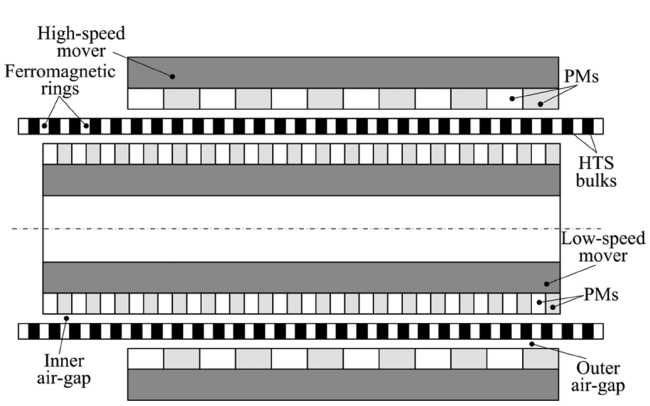

Fig. 5. Proposed tubular linear magnetic gear using HTS bulks.

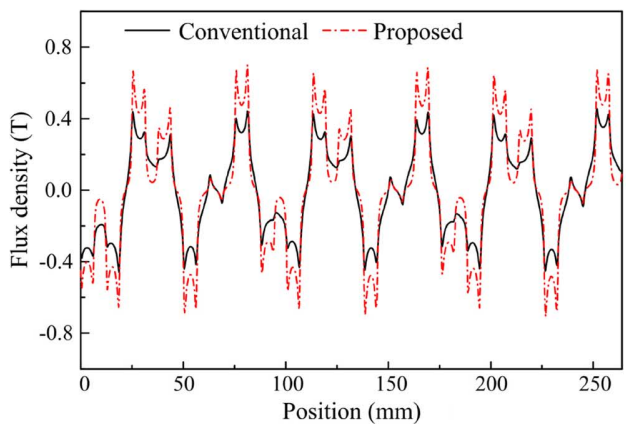

(a)

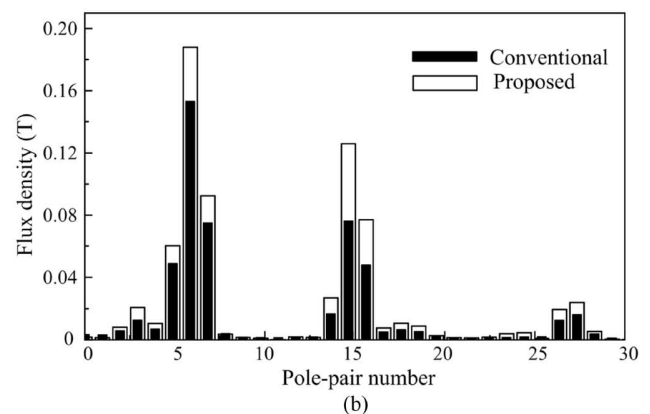

Fig. 6. Comparison of inner air-gap flux densities excited by PMs on highspeed mover. (a) Waveforms. (b) Spectra.

namely the HTS bulks are inserted between the ferromagnetic rings while they have the same pole-pitch. Fig. 5 shows the proposed gear, in which the HTS bulks are located in the stationary rings, thus facilitating the cooling arrangement. Also, it can achieve the thrust force density of $3.2 \mathrm{MN} / \mathrm{m}^{3}$.

Firstly, the inner air-gap flux densities of the two magnetic gears are analysed when only PMs on the high-speed mover serve as field excitation whereas PMs on the low-speed mover 


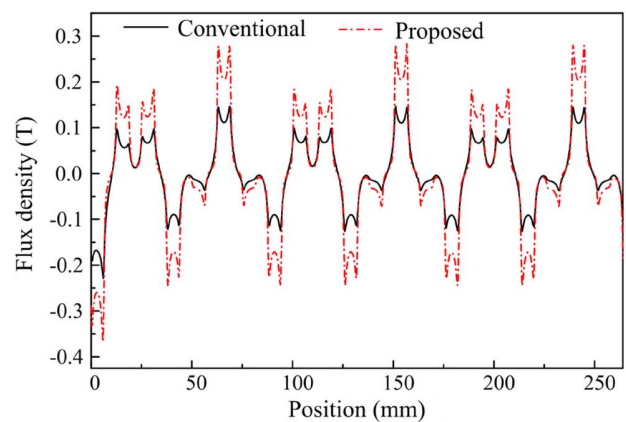

(a)

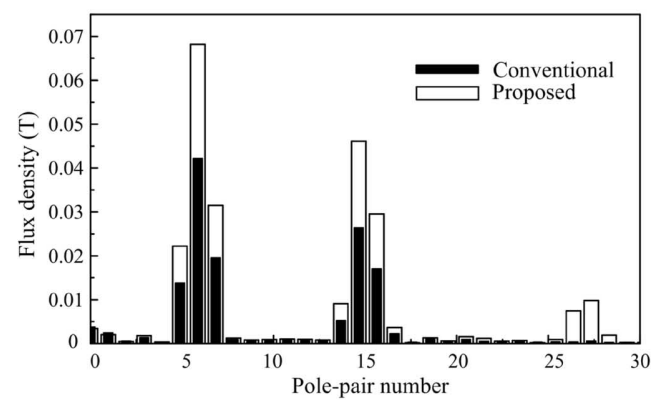

(b)

Fig. 7. Comparison of outer air-gap flux densities excited by PMs on low-speed mover. (a) Waveforms. (b) Spectra.

are set as air space. Fig. 6 shows the corresponding waveforms and harmonic spectra. It can be found that by using the HTS bulks, the amplitude of air-gap flux density can be improved greatly. By using spectrum analysis, it can also be found that the 6th and 15th harmonic components improve dramatically by using the HTS bulks. The largest asynchronous space harmonic which has 15 pole-pairs interacts with the 15 pole-pair number PMs on the low-speed mover, hence developing the desired steady thrust force.

Secondly, the outer air-gap densities are analysed when only PMs on the low-speed mover serve as excitation whereas PMs on the high-speed mover are set as air space. Fig. 7 shows their waveforms and spectra. It can be also found that the 6th and 15 th harmonic components improve greatly. The corresponding largest asynchronous space harmonic which has 6 pole-pairs interacts with the 6 pole-pair number PMs on the high-speed mover, hence developing the desired thrust force.

Thirdly, when their low-speed movers travel at $1 \mathrm{~m} / \mathrm{s}$ while their high-speed movers are fixed, the static thrust force characteristics of the low-speed mover are analysed. As shown in Fig. 8, the maximum thrust force of the proposed magnetic gear is improved by 1.8 times than that of the conventional one, which agrees with the theoretical 2.1 times as predicted by (8).

Finally, the thrust force transmission capacity of the proposed magnetic gear is obtained as shown in Fig. 9, which confirms that it can offer the force amplification by 2.5 times.

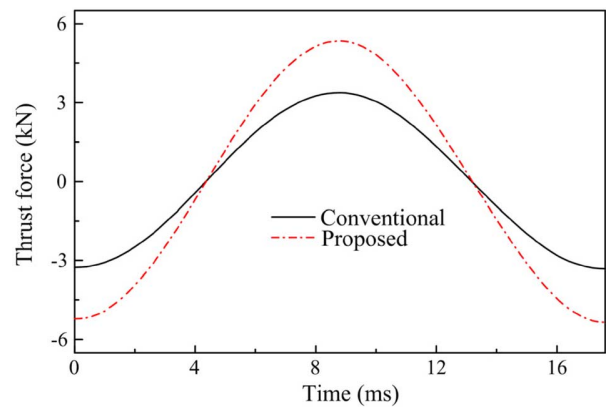

Fig. 8. Comparison of static thrust force characteristic of low-speed mover.

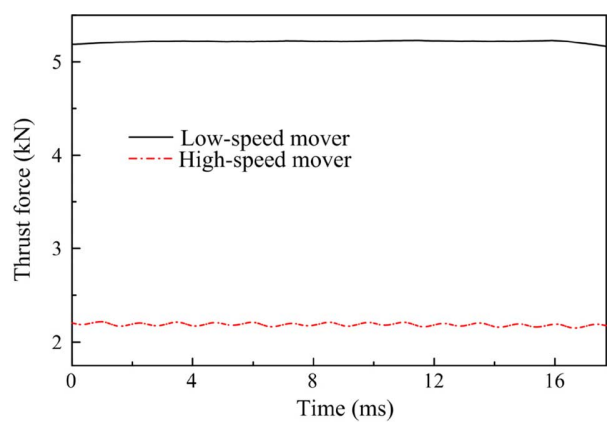

Fig. 9. Thrust force transmission capacity of proposed magnetic gear.

\section{CONCLUSION}

A new tubular linear magnetic gear using HTS bulks for field modulation has been proposed. By using magnetic circuit modeling, the corresponding air-gap flux density and developed thrust force are analytically derived, which is essential to provide physical insight for machine design. Also, by using finite element analysis, the use of HTS bulks to greatly improve the thrust force density is verified.

\section{REFERENCES}

[1] K. T. Chau, D. Zhang, J. Z. Jiang, C. Liu, and Y. J. Zhang, "Design of a magnetic-geared outer-rotor permanent-magnet brushless motor for electric vehicles," IEEE Transactions on Magnetics, vol. 43, no. 6, pp. 2504-2506, 2007.

[2] L. Jian, K. T. Chau, and J. Z. Jiang, "A magnetic-geared outer-rotor permanent-magnet brushless machine for wind power generation," IEEE Transactions on Industry Applications, vol. 45, no. 3, pp. 954-962, 2009.

[3] K. Atallah, J. Wang, and D. Howe, "A high-performance linear magnetic gear," Journal of Applied Physics, vol. 97, no. 10, pp. 10N516:1-10N516:3, 2005.

[4] K. T. Chau, D. Zhang, J. Z. Jiang, and L. Jian, "Transient analysis of coaxial magnetic gears using finite element comodeling," Journal of Applied Physics, vol. 103, no. 7, pp. 07F101: 1-07F101: 3, 2008.

[5] X. Liu, K. T. Chau, J. Z. Jiang, and C. Yu, "Design and analysis of interior-magnet outer-rotor concentric magnetic gears," Journal of Applied Physics, vol. 105, no. 7, pp. 1-3, 2009paper no., 07F101.

[6] L. Jian, K. T. Chau, W. Li, and J. Li, "A novel coaxial magnetic gear using bulk HTS for industrial applications," IEEE Transactions on Applied Superconductivity, vol. 20, no. 3, pp. 981-984, 2010.

[7] G. Krabbes, G. Fuchs, W.-R. Canders, H. May, and R. Palka, High Temperature Superconductor Bulk Materials. Weinheim: Wiley-VCH Verlag GmbH \& Co. KGaA, 2006. 